\title{
Unusual case of ATTR amyloidosis with cardiac manifestation and situs inversus totalis
}

\author{
Max Fritschka ${ }^{2,3}\left(\mathbb{D} \cdot\right.$ Michael Schlegl $^{4} \cdot$ Adrian Borges $^{5} \cdot$ Mathias Werner $^{6} \cdot$ \\ Rolf Gebker $^{2,3} \cdot$ Burkert Pieske ${ }^{1,2,3} \cdot$ Sebastian Kelle ${ }^{2,3}$
}

Received: 27 October 2016/Accepted: 16 December 2016/Published online: 23 January 2017

(C) The Author(s) 2017. This article is published with open access at Springerlink.com

\section{Sirs:}

Dextrocardia is a rare condition and occurs in approximately $0.01 \%$ of the general population [1]. The incidence of amyloidosis is estimated at between 5 and 13 per million inhabitants per year [2]. Cardiac involvement occurs in primary (amyloid light-chain, AL) amyloidosis (40-50\%), transthyretin (ATTR) amyloidosis (almost all cases) and in rare cases in secondary (amyloid A, AA) amyloidosis [3]. To our knowledge, the coincidence of situs inversus totalis and cardiac ATTR amyloidosis has never been reported before.

We are reporting the case of a 66-year-old male patient with known situs inversus totalis (Fig. 1a) and arterial hypertension who was hospitalized for right-sided angina pectoris with pain radiating into the neck and mandible alongside exertional dyspnea of NYHA class II. Laboratory results showed mildly positive troponin (high sensitivity):

Max Fritschka

fritschka@dhzb.de

1 Klinik für Innere Medizin mit Schwerpunkt Kardiologie, Campus Virchow-Klinikum, Charité-Universitätsmedizin Berlin, Augustenburger Platz 1, Berlin 13353, Germany

2 Klinik für Innere Medizin und Kardiologie, Deutsches Herzzentrum Berlin, Augustenburger Platz 1, 13353 Berlin, Germany

3 DZHK (German Centre for Cardiovascular Research), Partner Site Berlin, 652133, Berlin 13357, Germany

4 Praxis Westend, Cardiology Outpatient Clinic, Spandauer Damm 130, 14050 Berlin, Germany

5 HELIOS Klinikum Emil von Behring, Abteilung für Innere Medizin I / Kardiologie, Walterhöferstraße 11, Berlin 14165, Germany

6 HELIOS Klinikum Emil von Behring, Institut für Pathologie, Walterhöferstraße 11, Berlin 14165, Germany
24 ng/l; slightly elevated CK: 196 U/l; elevated NT-proBNP: $3496 \mathrm{ng} / \mathrm{l}$; CKD stage III, creatinine: $1.07 \mathrm{mg} / \mathrm{dl}$, GFR: $46 \mathrm{ml} / \mathrm{min}$ CKD-EPI. Urinalysis and other laboratory tests were without any significant pathological findings. The electrocardiogram showed sinus rhythm with low voltage in the limb leads (Fig. 2). A treadmill exercise test documented ventricular runs, triplets and multiple polymorphic extrasystoles under submaximal load without electrocardiographic ST-segment changes. The spirometry including body plethysmography and DLCO showed a restrictive ventilatory disturbance without pathological gas transfer. The transthoracic echocardiogram presented good left ventricular function (LVEF: 59\% biplane Simpson), slight hypokinesis in septal basal and medial segments and severe diastolic dysfunction of grade III with intermediate hypertrophy (IVSd: $15 \mathrm{~mm}$ ) (Fig. 3). The systolic pulmonary artery pressure was measured at $51 \mathrm{mmHg}(+\mathrm{CVP})$ indicating pulmonary hypertension. No significant valvular heart disease was found. Relative apical sparing was seen in the 2D strain analysis which led to the suspicion of cardiac amyloidosis. Cardiac catheterization (Fig. 1b, c) ruled out a coronary artery disease and a left ventricular biopsy was conducted to obtain endomyocardial tissue samples to confirm the diagnosis (Fig. 4). The pathohistological results were positive for a cardiac manifestation of ATTR amyloidosis. Congophilic material was detected in the congo red dye, which could be marked immunohistochemically with transthyretin antibodies. Finally, cardiac MRI could demonstrate the known situs inversus totalis and diffuse myocardial fibrosis (elevated T1-times of around $1400 \mathrm{~ms}$ measured with a 3.0 Tesla clinical cardiac MRI scanner) of the inferolateral (medial/basal) left ventricle typical of amyloidosis (Fig. 5). In view of these results, medical therapy with tafamidis, doxycycline (off-label), ursodeoxycholic acid, green tea (off-label) [4], a beta-blocker and diuretics was started. 

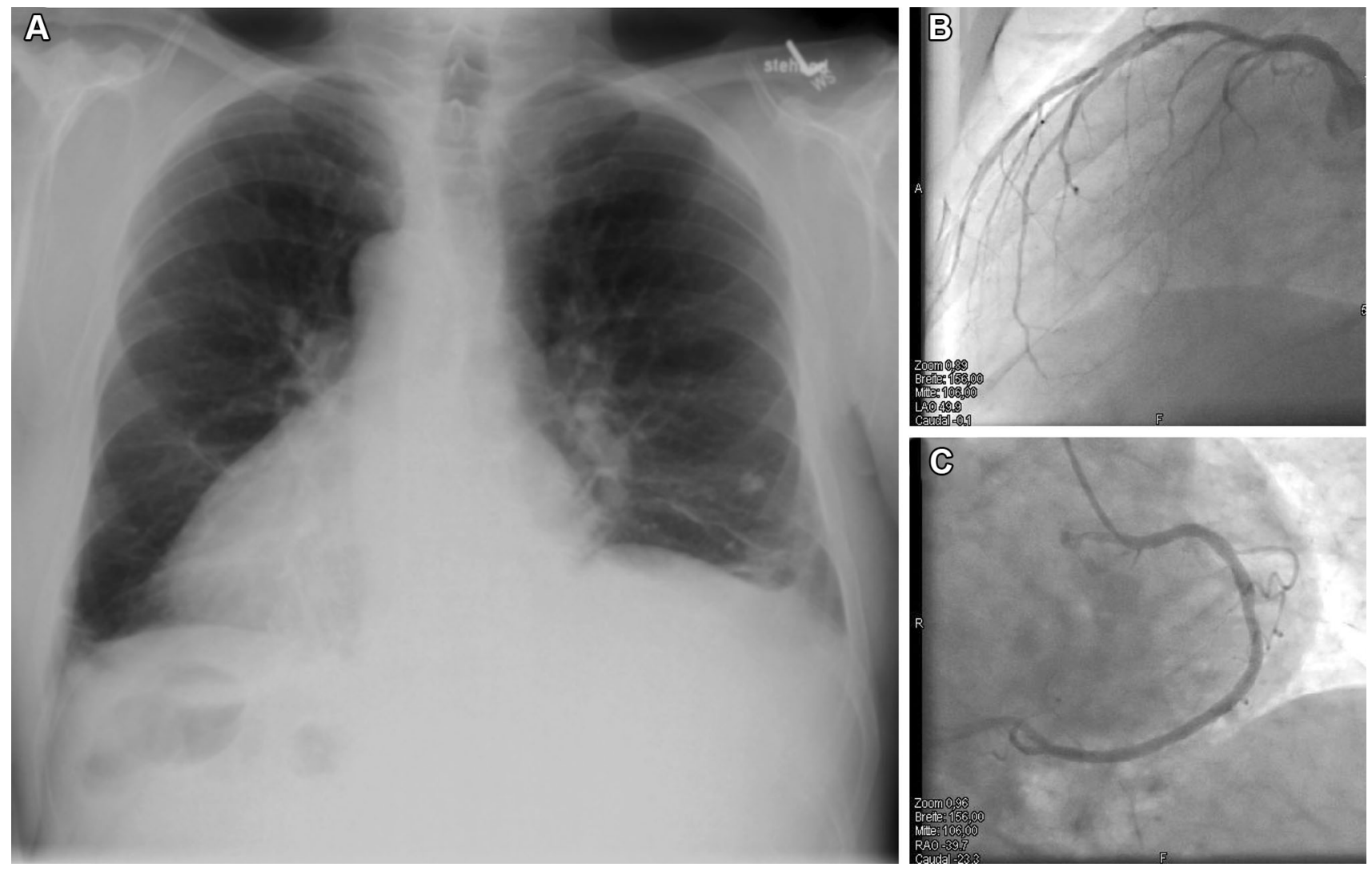

Fig. 1 a The chest radiograph demonstrates a situs inversus totalis with dextrocardia and right-sided aortic arch. b Angiography of the left coronary artery (LAO 49.9). c Angiography of the right coronary artery (RAO -39.7)

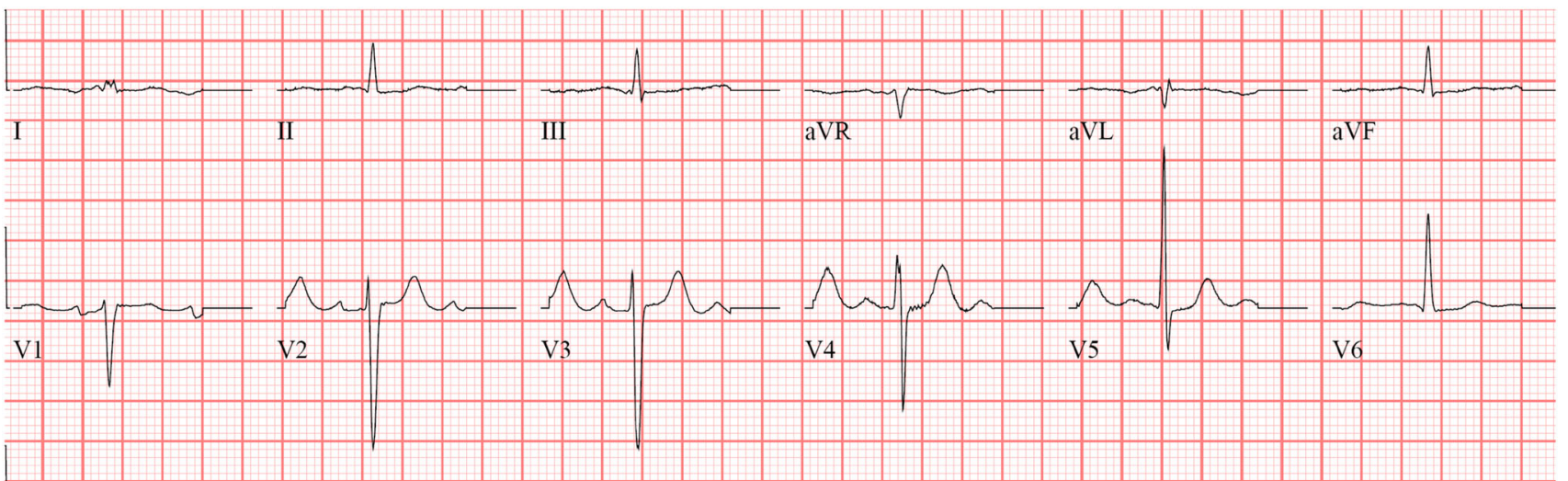

Fig. 2 The electrocardiogram shows a sinus rhythm with low voltages in the limb leads (typical for cardiac amyloidosis would be all limb leads $<5 \mathrm{~mm}$ in height). Further a typical slow R-progression as a pseudoinfarction pattern in the chest leads as well as a nonspecific intraventricular conduction delay is displayed 

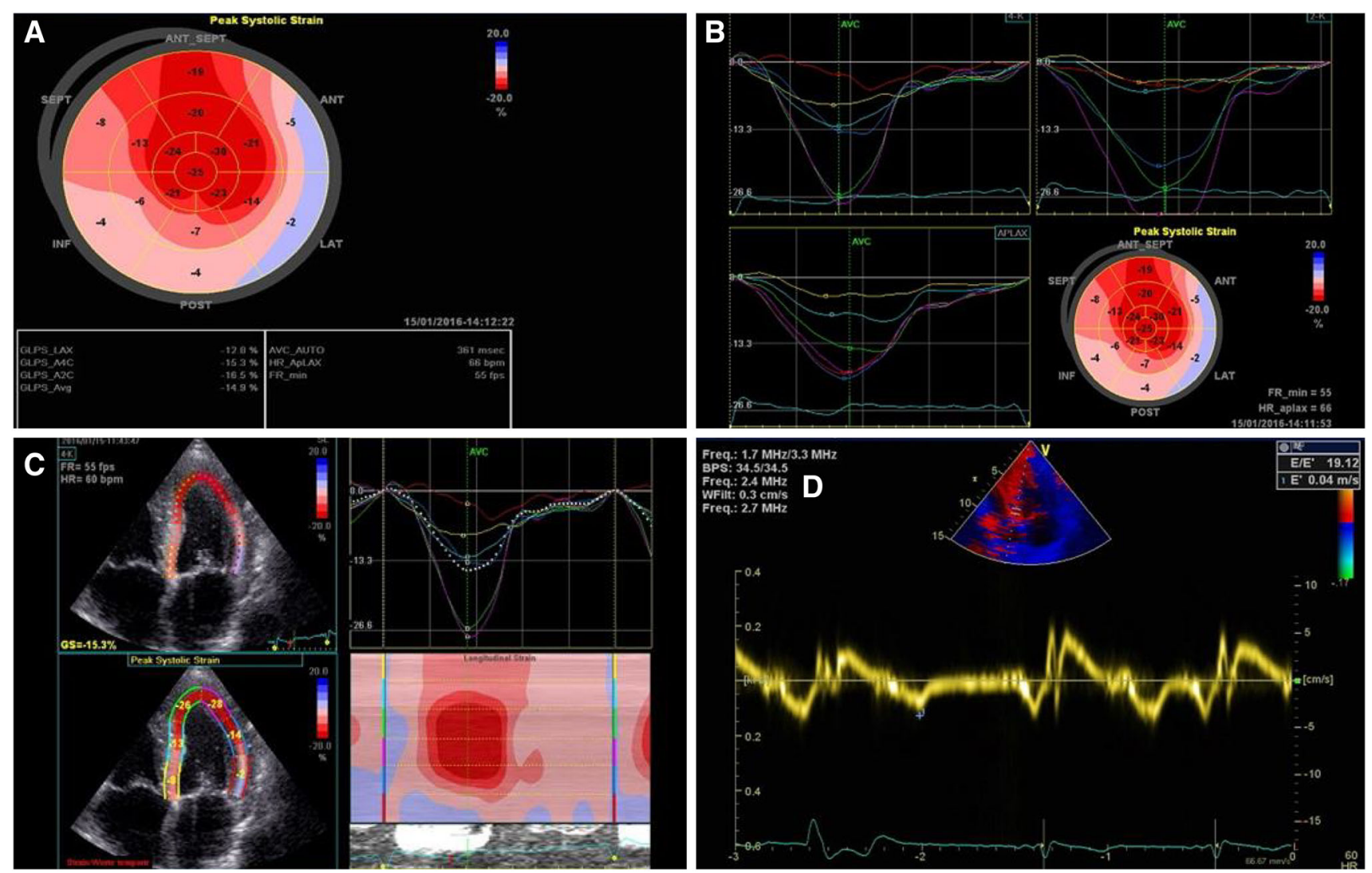

Fig. 3 a-c 2D strain analysis with apical sparing as a typical sign for amyloidosis. d Spectral tissue Doppler (TDI) shows an antegrade systolic and two retrograde waves $\left(E^{\prime}\right.$ and $\left.A^{\prime}\right)$. E/ $e^{\prime}$ measured 19.12 as an indication for a severe diastolic dysfunction

We report a rare case of ATTR amyloidosis with cardiac manifestation in a patient with situs inversus totalis. There seems to be no reported case of the coincidence of these two rare conditions to date. Yet cases of Kartagener syndrome (situs inversus, chronic sinusitis, and bronchiectasis due to primary ciliary dyskinesia) as an autosomal recessive disorder alongside renal amyloidosis are found in the current literature [5, 6]. Bronchiectasis is a known cause of secondary amyloidosis [7-9]. In this reported case no signs of proteinuria in the urine analysis or of bronchiectasis were found, with the spirometry showing a restrictive flow volume curve. Cardiac MRI, along with 2D strain evaluation conducted in transthoracic echocardiography or MRI [10] may lead to the non-invasive diagnosis of cardiac amyloidosis, with definite confirmation through endomyocardial biopsy. Yet a recent publication showed that today the diagnosis of ATTR amyloidosis could also be reached in patients without a monoclonal gammopathy using 

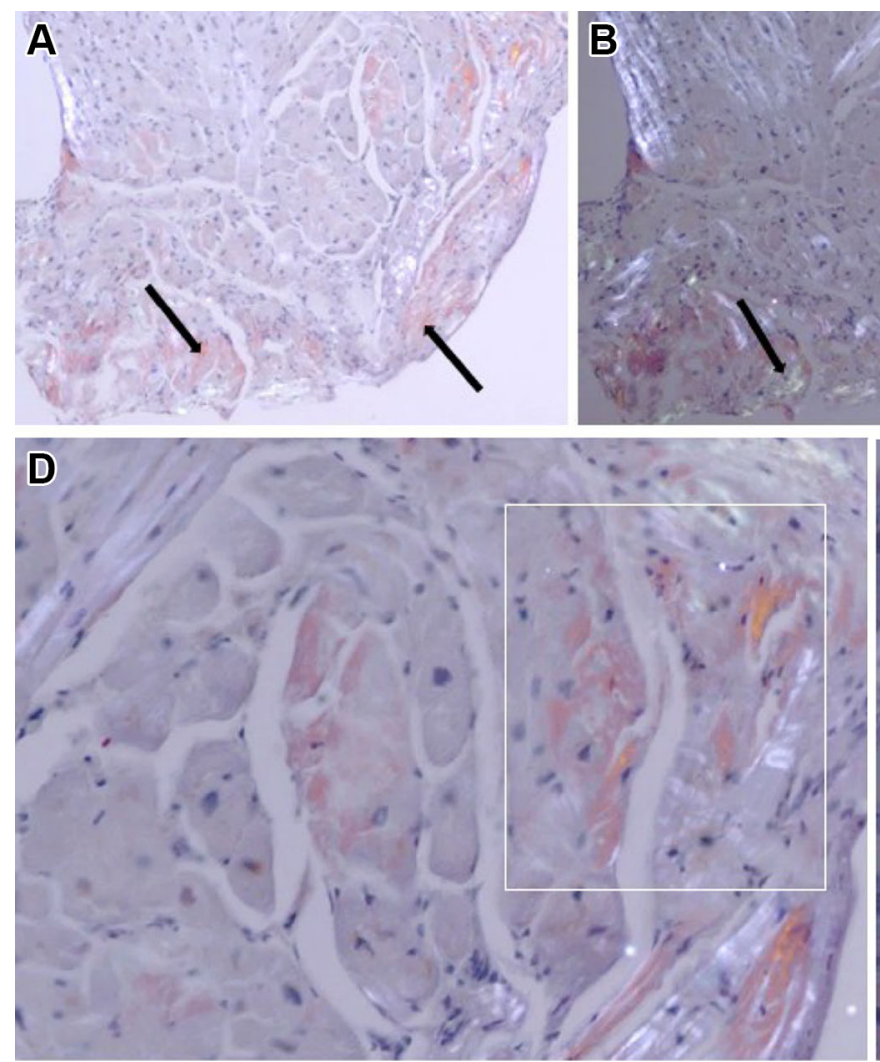

Fig. 4 a Myocardial biopsy with diffusely spread congophilic material. b Red congophilic material turns apple green in color on polarizing light. c Red congophilic material marked by an ATTR
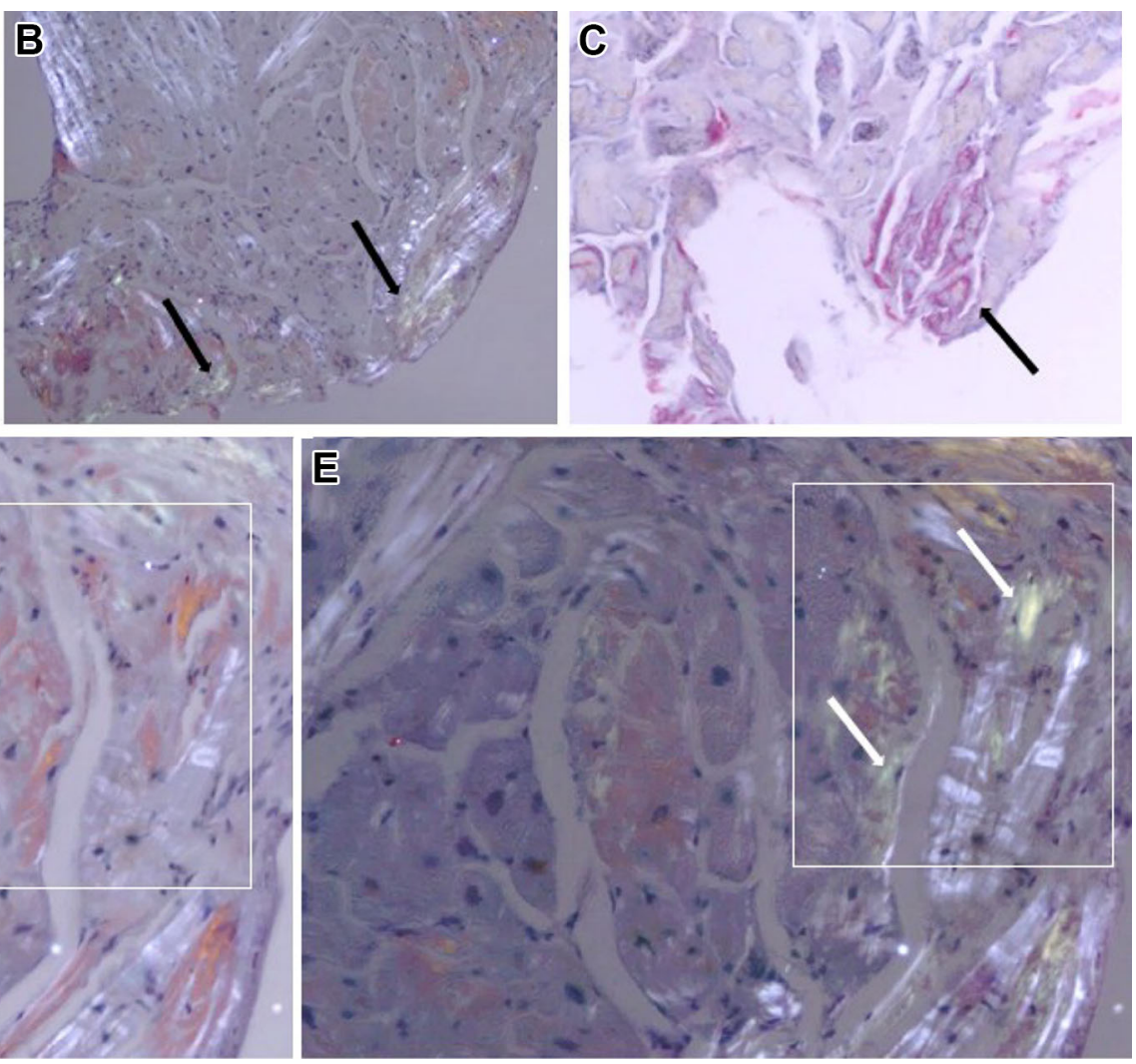

antibody. d Apple green birefringence demonstrated by congophilic amyloid fibrils on polarizing light microscopy in cardiac biopsy specimen. e Collagen shows a shining white appearance 

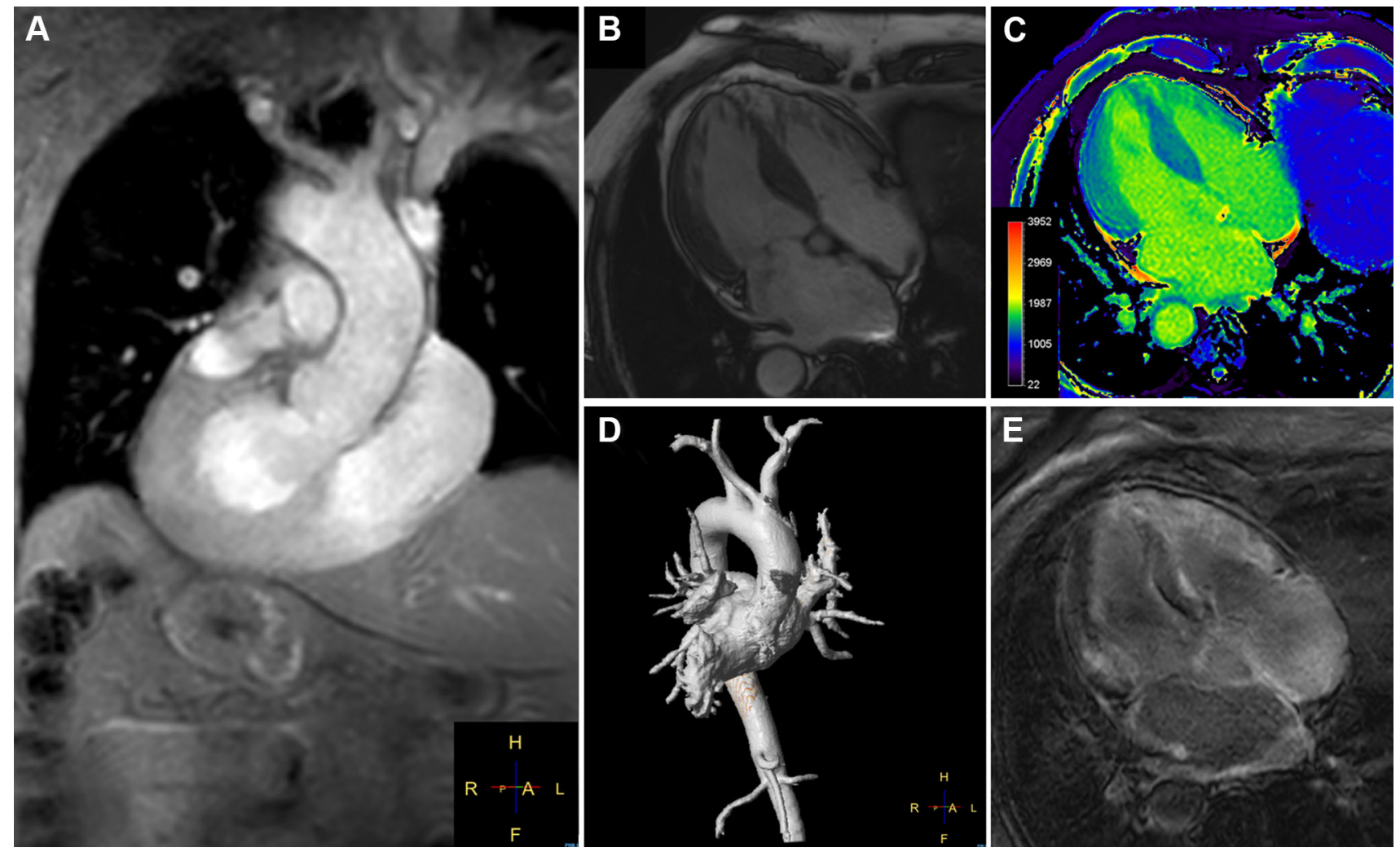

Fig. 5 a Images from a cardiac MRI confirms a situs inversus with dextrocardia. The outflow tract of the left ventricle, the left atrium and the right-sided aortic arch are displayed. b Four-chamber view with the mirrored left and right ventricle and thickened left ventricular wall. c Contrast-free T1-mapping reveals elevated values for T1-times

clinical information and bone scintigraphy alone [11], which might make a biopsy for diagnosis dispensable in certain cases in the future.

Acknowledgements Sebastian Kelle was supported by an unrestricted research grant from Philips Healthcare and received funding from the German Center for Cardiovascular Research (DZHK).

\section{Compliance with ethical standards}

Conflict of interest There is no conflict of interest to declare with regard to this publication.

Open Access This article is distributed under the terms of the Creative Commons Attribution 4.0 International License (http://crea tivecommons.org/licenses/by/4.0/), which permits unrestricted use, distribution, and reproduction in any medium, provided you give appropriate credit to the original author(s) and the source, provide a link to the Creative Commons license, and indicate if changes were made.

\section{References}

1. Evans WN, Acherman RJ, Collazos JC, Castillo WJ, Rollins RC, Kip KT et al (2010) Dextrocardia: practical clinical points and comments on terminology. Pediatr Cardiol 31(1):1-6 indicating increased amount of diffuse myocardial fibrosis. $\mathbf{d}$ Threedimensional reconstruction shows the aorta and pulmonary trunk. e Late gadolinium enhancement of the left ventricle demonstrates high signal intensity patterns, typical for a clinical picture of cardiac amyloidosis

2. Nienhuis HL, Bijzet J, Hazenberg BP (2016) The prevalence and management of systemic amyloidosis in western countries. Kidney Dis 2(1):10-19

3. Banypersad SM, Moon JC, Whelan C, Hawkins PN, Wechalekar AD (2012) Updates in cardiac amyloidosis: a review. J Am Heart Assoc 1(2):e000364

4. aus dem Siepen F, Buss SJ, Andre F, Seitz S, Giannitsis E, Steen $\mathrm{H}$ et al (2015) Extracellular remodeling in patients with wild-type amyloidosis consuming epigallocatechin-3-gallate: preliminary results of $\mathrm{T} 1$ mapping by cardiac magnetic resonance imaging in a small single center study. Clin Res Cardiol Off J Germ Cardiac Soc 104(8):640-647

5. El Houssni S, Laine M, Bziz A, Alhamany Z, Eddine Bourkadi J, Bayahia R (2015) Renal amyloidosis revealing a Kartagener's syndrome. Nephrol Ther 11(1):50-52

6. Katz M, Benzier E, Nangeroni L, Sussman B (1953) Kartagener's syndrome (situs inversus, bronchiectasis and chronic sinusitis). N Engl J Med 248(17):730-731

7. Edwards DF, Patton CS, Kennedy JR (1992) Primary ciliary dyskinesia in the dog. Probl Vet Med 4(2):291-319

8. Goldsmith DJ, Roberts IS, Short CD, Mallick NP (1996) Complete clinical remission and subsequent relapse of bronchiectasisrelated (AA) amyloid induced nephrotic syndrome. Nephron 74(3):572-576

9. Tuglular S, Yalcinkaya F, Paydas S, Oner A, Utas C, Bozfakioglu $S$ et al (2002) A retrospective analysis for aetiology and clinical findings of 287 secondary amyloidosis cases in Turkey. Nephrol Dial Transplant Off Publ Eur Dial Transpl Assoc Eur Renal Assoc 17(11):2003-2005 
10. Riffel JH, Keller MG, Aurich M, Sander Y, Andre F, Giusca S et al (2015) Assessment of global longitudinal strain using standardized myocardial deformation imaging: a modality independent software approach. Clin Res Cardiol Off J German Cardiac Soc 104(7):591-602
11. Gillmore JD, Maurer MS, Falk RH, Merlini G, Damy T, Dispenzieri A et al (2016) Nonbiopsy diagnosis of cardiac transthyretin amyloidosis. Circulation 133(24):2404-2412 